\title{
The Efficacy of Traditional Chinese Herbs Retention Enema Combined with Perineal Massage on Chronic Prostatitis/Chronic Pelvic Pain Syndrome (CP/CPPS)
}

\author{
Zhiyong $\mathrm{Wu}^{2, \dagger}$, Yingfan Zhang ${ }^{3, \dagger}$, Qiaohao $\mathrm{Hu}^{1, \dagger}$, Feng Zhou ${ }^{4}$, Yubao Wang ${ }^{5}$, Longwang Wang ${ }^{1, ~ *}$, \\ Ruixiang Song ${ }^{6}$, * \\ ${ }^{1}$ Department of Urology, The First Affiliated Hospital of Nanchang University, Nanchang, China \\ ${ }^{2}$ Shanghai Yubao Outpatient Department, Jing'anZiyuan Administrative Mansion, Shanghai, China \\ ${ }^{3}$ Department of Plastic and Reconstructive Surgery, Shanghai Ninth People's Hospital, Shanghai Jiao Tong University School of Medicine, \\ Shanghai, China \\ ${ }^{4}$ Department of Plastic and Reconstructive Surgery, Tongji Hospital, Tongji University, Shanghai, China \\ ${ }^{5}$ Shanghai Institute Of Planned Parenthood Research (SIPPR), Shanghai, China \\ ${ }^{6}$ Department of Urology, The First Affiliated Hospital of Naval Medical University, Shanghai, China
}

Email address:

360817782@qq.com (Ruixiang Song), 252423414@qq.com (Longwang Wang)

${ }^{*}$ Corresponding author

$\dagger$ Zhiyong Wu, Yingfan Zhang and Qiaohao Hu are co-first authors.

\section{To cite this article:}

Zhiyong Wu, Yingfan Zhang, Qiaohao Hu, Feng Zhou, Yubao Wang, Ruixiang Song, Longwang Wang. The Efficacy of Traditional Chinese Herbs Retention Enema Combined with Perineal Massage on Chronic Prostatitis/Chronic Pelvic Pain Syndrome (CP/CPPS). International Journal of Clinical and Experimental Medical Sciences. Vol. 7, No. 2, 2021, pp. 44-49. doi: 10.11648/j.ijcems.20210702.12

Received: March 18, 2021; Accepted: April 12, 2021; Published: April 24, 2021

\begin{abstract}
Purpose: To explore the clinical effect of traditional Chinese medicine retention enema combined with penis massage in the treatment of chronic prostatitis complicated with sexual dysfunction. Materials and methods: 200 patients with chronic prostatitis complicated with sexual dysfunction were randomly divided into two groups, 100 cases in each group. The combined treatment group was treated with traditional Chinese medicine retention enema combined with penis massage, and the control group was treated with traditional Chinese medicine retention enema alone. National Institutes of Health Chronic Prostatitis Symptom Index (NIH-CPSI), Chronic Prostatitis Related Sexual Function Index (CPSFI), International Index of Erectile Function-5 (IIEF-5), Chinese Index of Sexual Function for Premature Ejaculation (CIPE), Hamilton Anxiety Scale (HAMA) and Hamilton Depression Rating Scale (HAMD) and Routine examination of prostatic secretions (EPS) were used to evaluate the clinical effect before and after treatment. Results: The effective rate of the combined treatment group was significantly higher than that of the simple traditional Chinese medicine enema group. The total effective rate was $90 \%$ in the combined treatment group and $67 \%$ in the control group. The combined treatment group was significantly higher than the control group, and the difference was statistically significant $(\mathrm{P}<0.05)$. The scores of NIH-CPSI, IIEF-5, CIPE-5, HAMA and HAMD in the observation group were significantly lower than those in the control group, and the differences were statistically significant $(\mathrm{P}<0.05)$. Conclusion: Traditional Chinese medicine retention enema combined with penile massage for chronic prostatitis combined with ED can significantly improve the symptoms associated with chronic prostatitis, and can also significantly improve the patient's sexual function.
\end{abstract}

Keywords: Chronic Prostatitis/Chronic Pelvic Pain Syndrome, Traditional Chinese Herbs Retention Enema, Perineal Massage, Treatment Outcome 


\section{Introduction}

Chronic prostatitis/chronic pelvic pain syndrome ( $\mathrm{CP} / \mathrm{CPPS})$ is one of the common diseases in male genitourinary system, which is mainly described by pain or discomfort in the pelvic region, lasting for more than 3 months. It may also be accompanied with varying degrees of urinary irritation and sexual dysfunction, which seriously affect the life quality of the patients.

To date, the therapeutic interventions for CP/CPPS mainly include alpha-blockers, antibiotics, pain medications and multimodal therapy [1-3]. However, none of them could be defined as established treatments to alleviate the symptoms.

Chinese traditional herb has been widely used for the treatment of various diseases in China. And some Chinese medicine formulaes have been proved to be effective in CP/CPPS [4] According to our clinical experience, we use traditional Chinese herbs enema combined with perineal massage, and have achieved good results in the treatment of $\mathrm{CP} / \mathrm{CPPS}$. The specific herbal formulae includes dandelion, succulent sauce, salvia, and sanling, and these herbals are considered to alleviate pain, disperse inflammation, clear heat and activate blood circulation.

In this study, we conducted a retrospective research, in order to further investigate the effectiveness of our specific Chinese traditional treatment on the CP/CPPS symtoms. This retrospective study was approved by the ethics committee of Navy Medicine University.

\section{Patients and Treatments}

\subsection{Patients}

From September 2018 to July 2019, a total of 200 patients with $\mathrm{CP} / \mathrm{CPPS}$ were enrolled in our hospital and received our specific Chinese traditional medicine therapy. The CP/CPPS diagnosis is based on the following conditions: the National Institutes of Health (NIH) developed Chronic Prostatitis Syndrome Index $(\mathrm{NIH}-\mathrm{CPSI})>10$ points, white blood cells (WBCs) in prostatic fluid routine test $>10 / \mathrm{hp}$, reduced or disappeared lecithin bodies. And the course of the disease should be more than 3 months. Patients with benign prostatic hyperplasia $(\mathrm{BPH})$, acute bacterial prostatitis, uncontrolled diabetes mellitus, or poor general conditions were excluded from the study.

\subsection{Chinese Herbs Retention Enema}

The herbal formulae was consisted of dandelion, succulent sauce, big blood circulation, sanling, sputum, salvia, etc. Preparation process of the herbs included immersing for $3 \mathrm{~h}$, simmering for $1 \mathrm{~h}$, thickly frying and cooling, to get $120-150 \mathrm{ml}$ herb liquor whose temperature was $42^{\circ} \mathrm{C}$.

Before retention enema, the patients would be suggested to urinate and defecate and lie knee-chest position to expose the anus. The herb liquor was transported with a sterile infusion tube, dropped quickly, and the process was finished within 5 minutes. To maximize the effects, it was better to keep the herb liquor in the rectum for more than 4 hours.



Figure 1. Reflecting Regions in the Penile: As the theory of Chinese traditional medicine, there are several reflecting regions in the penile. And as showed in the figure, the reflecting region regarding to the prostate is in the glans. Pressing this region would be beneficial to prostate.

\subsection{Penile Massage}

Patients were maintained at supine position, keeping penile erection with the help of visual stimulation. If the patient suffers from erectile dysfunction, take a tablet of sildenafil 1 hour before treatment. All the procedures were performed in a manner that ensures patient privacy. Procedures of penile massage were depicted briefly as follows and there flecting regions in the penile were shown in Figure 1 and the procedures were depicted as follows:

1) The back of the penis were massaged with the thumb for 30 seconds and the massage strength should be comfortable for the patients;

2) The laterals and dorsum of the phallo some were massaged with the thumb from the root to the glans massage for about 1 minute respectively;

3) The frenum of the penis was massaged with the thumb for about 1 minute;

4) The penis was stimulated with the right hand of the clinician. And after erection, the phallo some was held and secured at the coronal groove with the left hand, then massaged with rotation clockwise;

5) The top of the glans was pressed gentally with the bended palm of clinician's right hand for about 1 minute. In order to congest and then diasole the prostate, accelerating the local blood circulation to promote the absorption of the herbal liquid, the clinician should make the penis soft and inhibit the ejaculation impulse which was less strong;

6) The procedures mentioned above cycle would be repeated 4-5 times for about 20 minutes to accumulate ejaculation impulse and make the penis full erection with perfect hardness;

7) After that, the penis would be stimulated and strong ejaculation impulse would be formed to achieve strong and powerful ejaculation. 
The penile massage was aimed to eject prostatic fluid as much as possible and to promote the discharge of inflammatory substances. And the whole therapy was given 1 time/d for continuous 7 days. During the treatment period, patients should avoid alcohol, tobacco, sexual life, full bladder and sedentariness. It was also suggested to exercise appropriately and avoid fatigue.

\subsection{Efficacy Judgment}

\subsubsection{NIH-CPSI}

The NIH-CPSI provides a reliable and valid assessment tool in the management of men with $\mathrm{CP} / \mathrm{CPPS}$, which is also commonly used clinically to evaluate the treatment effect. It is consisted of three aspects of the symptoms, namely pain and discomfort scores, urinary symptom scores and the impacts on life quality. And the higher of the total scores, the more serious conditions it represents.

Before and 12 weeks after therapy, NIH-CPSI of the patients was recorded and NIH-CPSI pain sub-score, NIH-CPSI urinary sub-score and NIH-CPSI QoL sub-score were analyzed as well.

The efficacy was judged by the changes between NIH-CPSI before and after therapy. Decrease by $>80 \%, 60-80 \%, 30-60 \%$ and $<30 \%$ represent significantly effective, effective, slightly effective and ineffective respectively.

\subsubsection{Evaluation of Negative Emotion}

Hamilton Depression (HAMD) and Anxiety (HAMA) scales [8] was used to evaluate the degree of patients' depression and anxiety respectively both before and after the therapy. And higher scores represent more serious conditions of the negative emotion.

\subsubsection{Prostatic Fluid Test}

The prostatic fluid was taken before and 12 weeks after treatment for examination. Microscopy, as well as count of leukocyte and lecithin corpuscle, was mainly performed.

\subsubsection{Satisfaction of Patients}

Improvement in patients' subjective feeling was also one of the indicators to determine the efficacy of the therapy, which included moist feeling of scrotum, discomfort in perineum, condition of erection and sexual satisfaction.

\subsection{Statistical Analysis}

The observed data were analyzed by statistical software SPSS 17.0. The measurement data were analyzed by t test, and the count data were analyzed by $\chi^{2}$ test. The difference was statistically significant at $\mathrm{P}<0.05$.

\section{Results}

A total of 200 male patients who suffered with CP/CPPS were included in the study. All the patients or their families have signed informed consent to exclude drug allergy, cognitive dysfunction, Parkinson's, mental illness, diabetes, severe heart and lung and kidney dysfunction.

The age ranged from 24 to 51 years, with an average of $34.57 \pm 3.48$ years. The course of disease was 6 to 31 months, with an average of $14.45 \pm 3.68$ months.

\subsection{NIH-CPSI and I Efficacy Evaluation}

Before and after treatment, the total NIH-CPSI, NIH-CPSI pain sub-score, NIH-CPSI urinary sub-score and NIH-CPSI QoL sub-score were recorded. After treatment, the score of pain, urination, and quality of life of the patients were significantly reduced $(\mathrm{p}<0.05)$, see Table 1 .

The average of the total CPSI of the patients after treatment was reduced to $4.88 \pm 2.32$ while the value before treatment was $24.07 \pm 7.56$, reduced by $67.07 \%$. The pain sub-score, NIH-CPSI urinary sub-score and NIH-CPSI QoL sub-score before treatment were $11.01 \pm 5.77,6.00 \pm 2.63$ and $7.06 \pm 3.25$. After therapy, the values reduced to $2.90 \pm 1.83,1.98 \pm 1.38$ and $2.19 \pm 1.41$ respectively, see table 1 .

Table 1. NIH-CPSI and Sub-score Before and 12 Weeks After Therapy.

\begin{tabular}{lllll}
\hline & Before Therapy & 12 Weeks After Therapy & P Value & Percentage Decline \\
\hline NIH-CPSI & $24.07 \pm 7.56$ & $4.88 \pm 2.32$ & $<0.01$ & $67.07 \%$ \\
NIH-CPSI pain sub-score & $11.01 \pm 5.77$ & $2.90 \pm 1.83$ & $<0.01$ & $62.49 \%$ \\
NIH-CPSI urinary sub-score & $6.00 \pm 2.63$ & $1.98 \pm 1.38$ & $<0.01$ & $58.50 \%$ \\
NIH-CPSIQoL sub-score & $7.06 \pm 3.25$ & $2.19 \pm 1.41$ & $<0.01$ & $65.75 \%$ \\
\hline
\end{tabular}

Among the patients, 51 of the 200 patients (25.5\%) had a reduction of NIH-CPSI by more than $80 \%$, which was evaluated as significantly effective. $95(47.5 \%)$ and $42(21.0 \%)$ patients had a reduction by $60-80 \%$ and $30-60 \%$, which were remarked as effective and slightly effective respectively. 12 of the 200 patients $(6.0 \%)$ were judged as ineffective for the reduction of the NIH-CPSI was below $30 \%$. The overall efficiency of the treatment was $94.0 \%$, see Table 2 .

Table 2. Evaluation of Therapeutic Efficacy.

\begin{tabular}{lllll}
\hline Rank of Therapeutic Effect & Descend Range Percentage & n & Proportion & General Effective Rate \\
\hline Significantly Effective & $>80 \%$ & 51 & $25.5 \%$ & \\
Effective & $60-80 \%$ & 95 & $47.5 \%$ & $94 \%$ \\
Slightly Effective & $30-60 \%$ & 42 & $21.0 \%$ & \\
Ineffective & $<30 \%$ & 12 & $6.0 \%$ & \\
\hline
\end{tabular}




\subsection{Negative Emotion}

HAMA and HAMD were recorded before and 12 weeks after treatment to evaluate the negative emotion, including anxiety and depression. HAMA was reduced from $14.45 \pm 6.55$ to $4.39 \pm 3.74(<0.01)$, while HAMD was reduced from $4.39 \pm 3.74$ to $6.99 \pm 5.91(<0.01)$, see Table 3 . The mental state of most patients also improved significantly.

Table 3. Evaluation of Patients 'Negitive Emotions Before and 12 Weeks After Therapy.

\begin{tabular}{llll}
\hline & Before Therapy & 12 Weeks After Therapy & P Value \\
\hline HAMD & $15.76 \pm 9.39$ & $6.99 \pm 5.91$ & $<0.01$ \\
HAMA & $14.45 \pm 6.55$ & $4.39 \pm 3.74$ & $<0.01$ \\
\hline
\end{tabular}

\subsection{Prostatic Fluid}

The prostatic fluid of patients before and after treatment was collected for direct microscopy. It was found that there was WBC accumulation in prostatic fluid before treatment, and the glandular fluid was clear after treatment without obvious pus accumulation. In prostatic fluid test, $\mathrm{WBC}<3$ and lecithin corpuscles were found in 198 of 200 patients.

\subsection{Subjective Symptoms}

Subjective symptoms of the patients including moist feeling of scrotum, discomfort in perineum, condition of erection and sexual satisfaction were also evaluated. Before treatment, 121 of the 200 patients suffered from moist feeling of scrotum. 132 of them suffered from discomfort in perineum. And declining in condition of erection and sexual satisfaction were seen in 113 and 107 patients. After treatment, the symptoms above were improved, see table 4 .

Table 4. Evaluation of Patients 'Subjective Symptoms.

\begin{tabular}{|c|c|c|c|c|}
\hline Subjective Symptom & $\begin{array}{l}\text { Number of Patients with } \\
\text { Symptoms before Therapy }\end{array}$ & $\begin{array}{l}\text { Proportion of } \\
\text { all patients }\end{array}$ & $\begin{array}{l}\text { Number of Patients with } \\
\text { Symptoms } 12 \text { weeks after therapy }\end{array}$ & $\begin{array}{l}\text { Improvement (Decline in } \\
\text { number) Rate }\end{array}$ \\
\hline Moist Feeling in Scrotum & 121 & $60.5 \%$ & 19 & $84.30 \%$ \\
\hline Discomfort in Perineum & 132 & $66.0 \%$ & 32 & $75.76 \%$ \\
\hline Decreased Condition of Erection & 113 & $56.5 \%$ & 17 & $84.96 \%$ \\
\hline Sexual Disatisfaction & 107 & $53.5 \%$ & 14 & $86.92 \%$ \\
\hline
\end{tabular}

\section{Discussion}

Chronic prostatitis is one of the common diseases in urology. It is estimated that about $50 \%$ of men have chronic prostatitis symptoms during their lifetime and the prevalence rate is $2.0 \% \sim 14.2 \%(5,6)$. Generally, the symptoms of chronic prostatitis include long-term, repeated pelvic area pain or discomfort, which is often accompanied by urinary symptoms and sexual dysfunction with different degrees.

Chronic prostatitis has a complex etiology and diverse pathogenesis [7]. At present, the comprehensive treatment methods including drug therapy, psychotherapy, physical therapy, and surgical treatment are used clinically. The main goal of treatment is to relieve pain, improve urinary symptoms and improve patients' quality of life [8]. In terms of drug therapy, the main choices are antibiotics, areceptor-blockers, 5-a reductase inhibitors and non-steroidal anti-inflammatory analgesics (NSAIDs).

However, although drugs have achieved certain effect, it is limited and always unsatisfactory to most patients. In other words, it is difficult to cure chronic prostatitis thoroughly [1, 5]. Clinical experience show that traditional drug treatment can only temporarily improve the related symptoms, but it can not fundamentally block the inflammatory process of chronic prostatitis. As the disease progresses, the effect of drug treatment becomes weaker and weaker, causing repeated episodes and prolonged illness, which brings serious physical and mental burden to the patients [9].

Modern medicine believes that the cause of chronic prostatitis is complicated. However, it has common features in pathology, which includes congestion and edema in prostate gland, acinar and mesenchyme, inflammatory cell infiltration, gland obstruction, gland fluid and inflammatory secretion retention, inflammation and interstitial fibrosis, prostate induration and sclerosis, glandular contraction [10].

We believe that dredging the channels and glandular veins, timely and effective discharge of stagnant gland fluid and inflammatory secretions, is the key to the treatment of chronic prostatitis. We applied traditional Chinese medicine to promote blood circulation, clearing away inflammatory substances and detoxification, reducing swelling and relieving pain, clearing sputum and removing phlegm as the basic treatment principle.

We used traditional Chinese medicine retention enema combined with penile massage to promote the discharge of glandular fluid and inflammatory secretions, which seemed to get a significant therapeutic effect. Modern pharmacological studies have confirmed that blood stasis and phlegm (TCM) can improve pelvic microcirculation, increase local blood perfusion, relieve inflammatory obstruction and promote absorption of inflammatory exudate. Furthermore, it can also improve immunity and stimulate proliferation of reticuloendothelial system, as well as promote phagocytosis of white blood cells [11-15].

In the prescription, Pugongying (Dandelion) is sweet and slightly bitter in flavor and cold in nature and is effective in clearing heat and detoxifying, and dissipating lump and mass. Baijiangcao (Patriniavillosa) is pungent in flavor and slight cold in nature, with the function of heat-clearing and detoxification, phlegm elimination and pus-discharging. Daxueteng (Stephania macrocephala) works to smooth meridians and collaterals, to promoting diuresis and relieve stranguria, and to alleviate stasis-induced pain. Sanling is 
pungent and bitter in flavor and warm in nature. It has the effects of promoting blood and Qi circulation, removing stagnation and relieving pain. Ezhu (Curcuma zedoary) is pungent and bitter in flavor and warm in nature with the function of removing blood stasis and relieving pain. Danshen (Salvia) is bitter in flavor and slight cold in nature with the function of unblocking meridians and relieving pain.

When given as retention enema, the temperature of Chinese herb liquid is 42 degrees to get a certain hyperthermia effect on the prostate, which is conducive to the regression of prostatic inflammation. As there are 2 to 6 small genital veins between the rectal vein and the bladder venous plexus [16], the herb absorbed by the rectum can directly enter the prostate tissue through the genital vein to avoid the "first pass effect" and gastrointestinal reaction of the liver. In addition, according to our clinical experience, the effect is better to that of oral medication.

In order to enhance the contractility of the prostate and the ejaculation ability of the patient, and effectively empty the prostate acinus and glandular duct, we have established a scientific and standardized penile massage method, combined with the advantages of traditional Chinese medicine retention enema, greatly improving the clinical treatment effect of chronic prostatitis. Penile massage can effectively promote blood circulation of the penis and prostate. The technique is both unique and novel, which can stimulate the prostate to secrete glandular fluid actively rather than passive secretion induced by prostate massage from the anus. It is believed by us that the method is more effective to empty the prostate than the previous ones.

After stimulating the penis erection, immediately we pressed the top of the glans to soften the penis and inhibit the ejaculation impulse of the patient, which is beneficial to increase the blood circulation of the prostate and promote the absorption of Chinese medicine. After repeating the aforementioned treatment protocol 4-5 times, the patient's penile erection is much more firmer and the ejaculation impulse is more intense.

At this time, the patient's prostate strongly contracts, achieving strong and powerful ejaculation, which is beneficial to the empty the inflammation and necrotic substances in the prostate gland. Excretion of multiple places, so that the prostate is truly empty, promote the prostate to soften, shrink the shape, reduce swelling and pain and accelerate the absorption of inflammation, effectively relieve the symptoms of perineal pain and discomfort caused by gland swellings. After the ejaculation, the prostate is relatively empty.

After that, 5-10ml urethral perfusion of levofloxacin is performed immediately, which is more conducive to the reflux of the drug into the prostate tissue. We believe that penile massage can make the prostate contraction strongly and achieve strong and powerful ejaculation, thus achieving the combined contraction and excretion of multiple organs such as the prostate, seminal vesicle, vas deferens and posterior urethra, which promotes all tubes more effectively than prostate massage and regular drainage.

Drainage of inflammatory substances in the cavity, dredge the prostate gland, reduce the accumulation of local harmful metabolites, reduce the tissue edema and metabolic disorders caused by the stimulation of inflammatory substances, promote prostate blood circulation, and its therapeutic effect is equivalent to surgical suppuration Incision and drainage of sexual lesions.

However, there are still several limitations in the novel therapy and this study. First, patients are needed to fully expose their genitals, which may be difficult to some of them. Then, it is neccessary to spend more time relatively to finish a single treatment, which seemed less efficient. Finally, the present study is an observational study and more researches are needed to further evaluate the efficacy of our novel method.

\section{Conclusion}

The application of traditional Chinese medicine retention enema combined with penile massage is a new method for the treatment of chronic prostatitis. After combined treatment, the symptoms of chronic prostatitis were significantly relieved, the scores of depression and anxiety were significantly reduced, and the quality of life of patients was significantly increased.

\section{Funding}

The study was supported by the Traditional Chinese Medicine Science and Technology Program of Jiangxi Province (No. 2020A0231).

\section{References}

[1] Sharp VJ, Takacs EB, Powell CR. Prostatitis: diagnosis and treatment. Am Fam Physician. 2010; 82 (4): 397-406.

[2] Rees J, Abrahams M, Doble A, Cooper A, Prostatitis Expert Reference G. Diagnosis and treatment of chronic bacterial prostatitis and chronic prostatitis/chronic pelvic pain syndrome: a consensus guideline. BJU Int. 2015; 116 (4): 509-25.

[3] Tran CN, Shoskes DA. Sexual dysfunction in chronic prostatitis/chronic pelvic pain syndrome. World J Urol. 2013; 31 (4): 741-6.

[4] Cui X, Naveed M, Baig M, Wang W, Mikrani R, Liu Z, et al. Therapeutic effects of Qianlie Tongli decoction on chronic prostatitis/chronic pelvic pain syndrome induced by peptide T2 in mice. J Pharm Pharmacol. 2020.

[5] Nickel JC, Shoskes DA, Wagenlehner FM. Management of chronic prostatitis/chronic pelvic pain syndrome (CP/CPPS): the studies, the evidence, and the impact. World J Urol. 2013; 31 (4): 747-53.

[6] McNaughton Collins M. The impact of chronic prostatitis/chronic pelvic pain syndrome on patients. World $\mathrm{J}$ Urol. 2003; 21 (2): 86-9.

[7] Anichkov NM, Kniaz'kin IV. [Chronic prostatitis: 1) etiology, pathogenesis, modeling, classification]. Adv Gerontol. 2003; 11: 84-92. 
[8] Zaidi N, Thomas D, Chughtai B. Management of Chronic Prostatitis (CP). Curr Urol Rep. 2018; 19 (11): 88.

[9] Shoskes DA. The challenge of erectile dysfunction in the man with chronic prostatitis/chronic pelvic pain syndrome. Curr Urol Rep. 2012; 13 (4): 263-7.

[10] Shoskes DA, Prots D, Karns J, Horhn J, Shoskes AC. Greater endothelial dysfunction and arterial stiffness in men with chronic prostatitis/chronic pelvic pain syndrome--a possible link to cardiovascular disease. J Urol. 2011; 186 (3): 907-10.

[11] Zhang Y, Liu Y. [Study on treatment of blood stasis syndrome of chronic prostatitis using prostatitis decoction and its extract capsule]. Zhongguo Zhong Xi Yi Jie He Za Zhi. 1998; 18 (9): 534-6.

[12] Xiong XJ, Wang Z, Wang J. Innovative Strategy in Treating Angina Pectoris with Chinese Patent Medicines by Promoting Blood Circulation and Removing Blood Stasis: Experience from Combination Therapy in Chinese Medicine. Curr Vasc Pharmacol. 2015; 13 (4): 540-53.

[13] Liu W, Yang S, Fu M, Li J, Song Y, Wei B, et al. Chinese patent medicine for chronic obstructive pulmonary disease based on principles of tonifying Qi, promoting blood circulation by removing blood stasis, and resolving phlegm: a systematic review of randomized controlled trials. J Tradit Chin Med. 2015; 35 (1): 1-10.

[14] Wang Z, Yuan L, Wang Y, Yang B, Dong X, Gao Z. Efficacy and safety of Chinese herbal medicine for chronic prostatitis associated with damp-heat and blood-stasis syndromes: a meta-analysis and literature review. Patient Prefer Adherence. 2016; 10: 1889-902.

[15] Lu M, Wang TY, Tian XX, Shi XH, Fan GW, Zhang Y, et al. [Interaction of anti-thrombotic and anti-inflammatory activities of commonly used traditional Chinese medicine for promoting blood circulation and removing blood stasis revealed by network pharmacology analysis]. Yao Xue Xue Bao. 2015; 50 (9): 1135-41.

[16] Shafik A. Anal submucosal injection: a new route for drug administration. VI. Chronic prostatitis: a new modality of treatment with report of eleven cases. Urology. 1991; 37 (1): $61-4$. 